Supporting Information

\title{
Cosolvent Effects on the aggregation and Micellar growth of Ester- Containing Gemini Surfactants
}

Soheila Javadian $^{\mathrm{a}^{*}}$, Shirin Lashgari ${ }^{\mathrm{a}}$, Jamal Kakemam ${ }^{\mathrm{a}}$, Hasti Aghdastinat ${ }^{\mathrm{a}}$, Ali R. Tehrani-Bagha ${ }^{\mathrm{b}} \quad$ •

${ }^{a}$ Department of Physical Chemistry, Tarbiat Modares University, P.O. Box 14115-117, Tehran, Iran.

${ }^{\mathrm{b}}$ Department of Chemical and Petroleum Engineering, American University of Beirut, PO Box 11236, Beirut, 1107-2020, Lebanon

*Email: javadian_s@modares.ac.ir, javadians@yahoo.com

Fax: +98-21-82883455 
Supporting Information

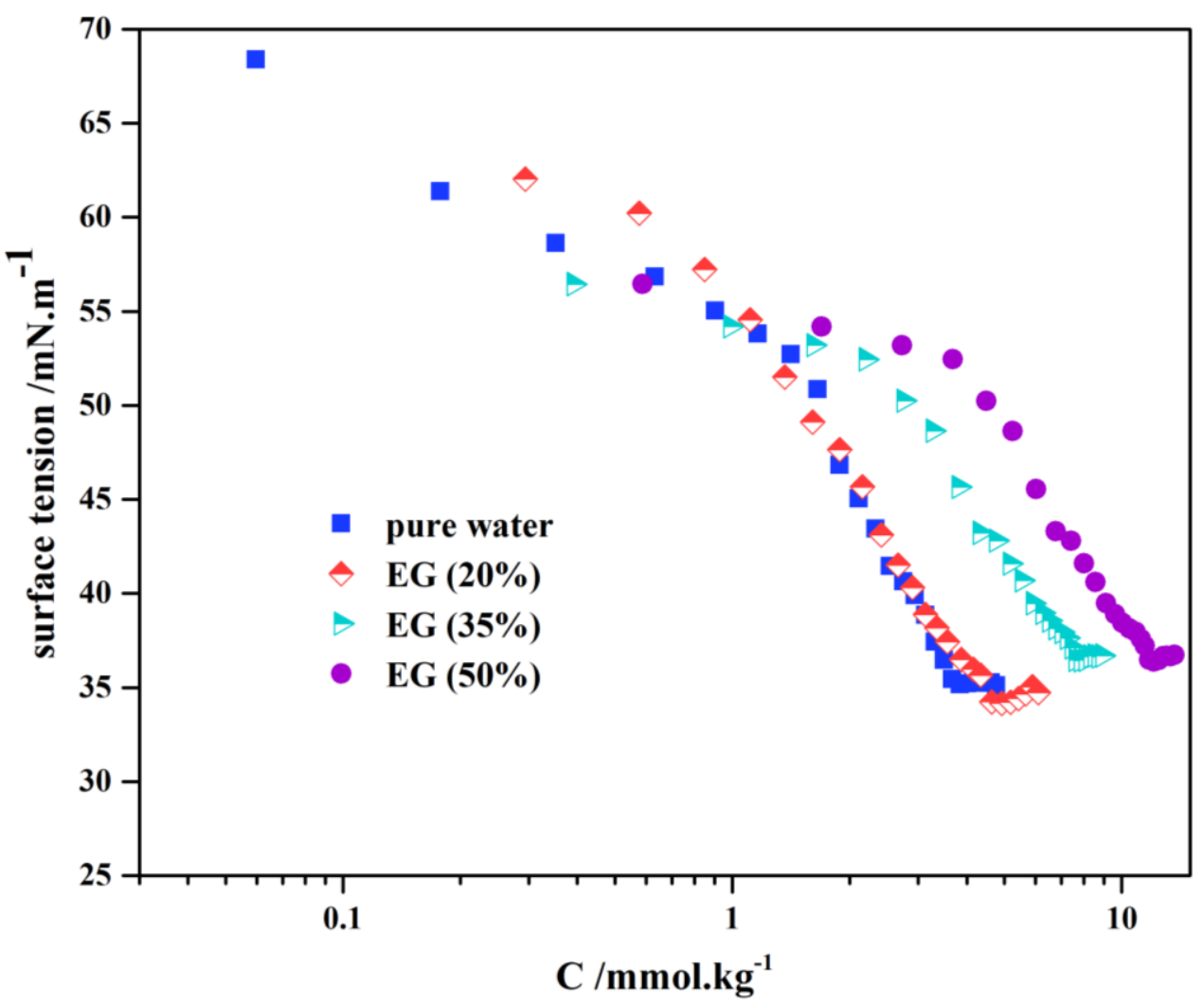

Figure S1. Surface tension vs. TTAB concentration at $298.15 \mathrm{~K}$ 
Supporting Information

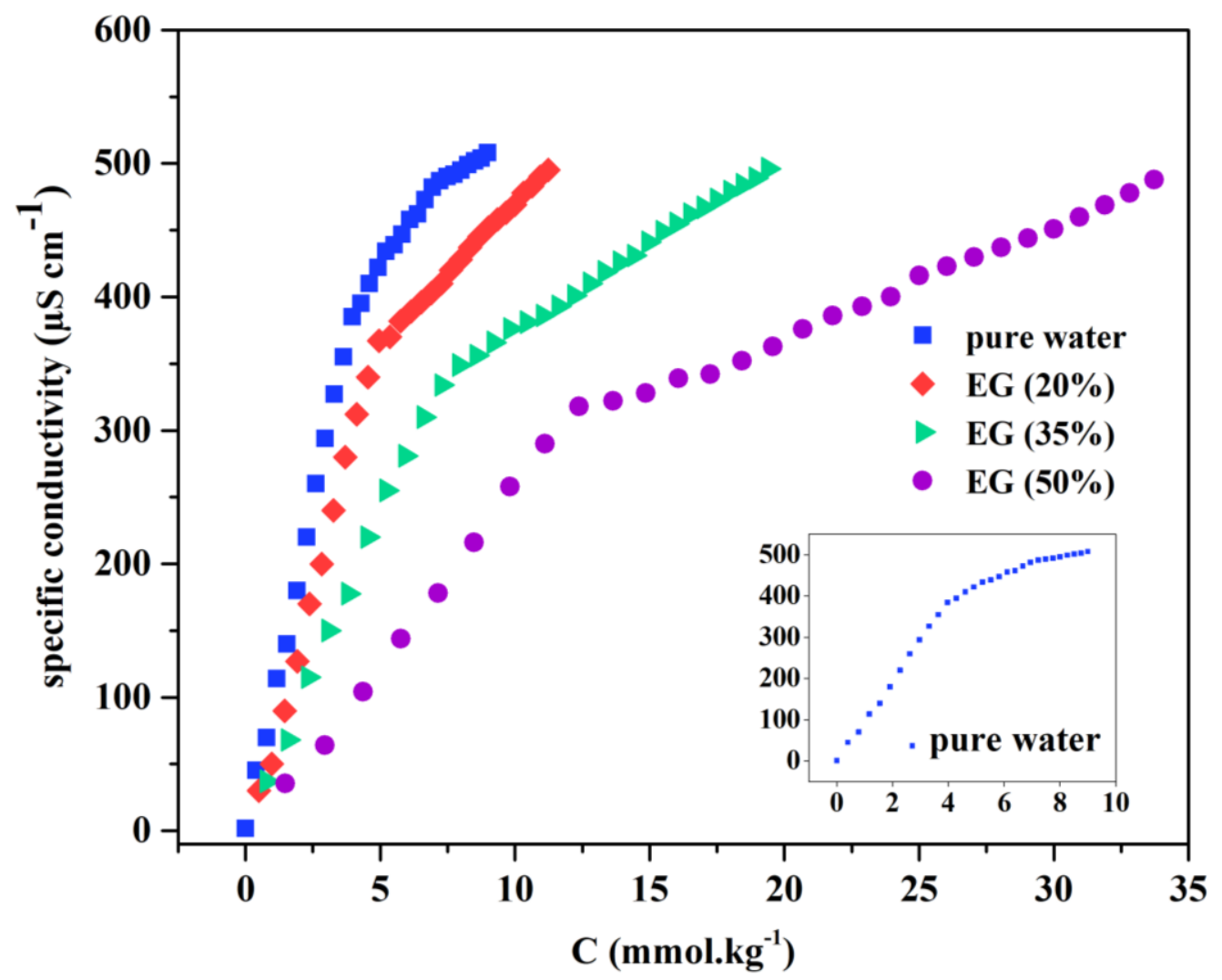

Figure 2. Specific conductivity vs. TTAB concentration at $298.15 \mathrm{~K}$ 
Supporting Information

Table S1. The surface tension $(\gamma)$ and corresponding concentrations $(C)$ of dodecyl esterquat gemini $(s=3)$, dodecyl betainate gemini $(s=3)$, and TTAB in the water-EG medium at $T=298.15 \mathrm{~K}$

\begin{tabular}{|c|c|c|c|c|c|c|c|}
\hline \multicolumn{8}{|c|}{ dodecyl esterquat gemini $(\mathrm{s}=3)$} \\
\hline \multicolumn{2}{|c|}{ EG $(0 \%)$} & \multicolumn{2}{|c|}{ EG $(20 \%)$} & \multicolumn{2}{|c|}{ EG $(35 \%)$} & \multicolumn{2}{|c|}{ EG (50\%) } \\
\hline $\begin{array}{c}C \\
\mathrm{mmol} \cdot \mathrm{kg}^{-1}\end{array}$ & $\begin{array}{c}\gamma \\
\mathrm{mN} \cdot \mathrm{m}^{-1}\end{array}$ & $\begin{array}{c}C \\
\mathrm{mmol} \cdot \mathrm{kg}^{-1}\end{array}$ & $\begin{array}{c}\gamma \\
\mathrm{mN} \cdot \mathrm{m}^{-1}\end{array}$ & $\begin{array}{c}C \\
\mathrm{mmol} \cdot \mathrm{kg}^{-1}\end{array}$ & $\begin{array}{c}\gamma \\
\mathrm{mN} \cdot \mathrm{m}^{-1}\end{array}$ & $\begin{array}{c}C \\
\mathrm{mmol} \cdot \mathrm{kg}^{-1}\end{array}$ & $\begin{array}{c}\gamma \\
\mathrm{mN} \cdot \mathrm{m}^{-1}\end{array}$ \\
\hline 0.036 & $58.06 \pm 0.02$ & 0.032 & $61.4 \pm 0.01$ & 0.117 & $54.86 \pm 0.02$ & 0.163 & $50.2 \pm 0.05$ \\
\hline 0.070 & $53.20 \pm 0.03$ & 0.064 & $58 \pm 0.04$ & 0.229 & $53.14 \pm 0.02$ & 0.318 & $49.8 \pm 0.03$ \\
\hline 0.104 & $50.42 \pm 0.04$ & 0.096 & $57.21 \pm 0.03$ & 0.336 & $52.84 \pm 0.05$ & 0.465 & $47.22 \pm 0.02$ \\
\hline 0.137 & $49.54 \pm 0.03$ & 0.129 & $55.89 \pm 0.04$ & 0.438 & $48.76 \pm 0.03$ & 0.604 & $46.11 \pm 0.04$ \\
\hline 0.170 & $48.13 \pm 0.03$ & 0.161 & $55.13 \pm 0.04$ & 0.536 & $46.13 \pm 0.03$ & 0.737 & $44.03 \pm 0.02$ \\
\hline 0.201 & $44.32 \pm 0.02$ & 0.193 & $52.16 \pm 0.02$ & 0.629 & $44.75 \pm 0.02$ & 0.863 & $43.98 \pm 0.04$ \\
\hline 0.232 & $40.65 \pm 0.02$ & 0.225 & $49.85 \pm 0.02$ & 0.719 & $42.72 \pm 0.03$ & 0.983 & $42.87 \pm 0.03$ \\
\hline 0.263 & $39.21 \pm 0.02$ & 0.257 & $48.14 \pm 0.03$ & 0.805 & $41.92 \pm 0.03$ & 1.098 & $41.19 \pm 0.02$ \\
\hline 0.292 & $37.6 \pm 0.02$ & 0.289 & $45.6 \pm 0.02$ & 0.888 & $42 \pm 0.001$ & 1.208 & $40.92 \pm 0.02$ \\
\hline 0.321 & $36.7 \pm 0.02$ & 0.321 & $44.2 \pm 0.03$ & 0.968 & $42.1 \pm 0.02$ & 1.313 & $40.02 \pm 0.02$ \\
\hline 0.350 & $35.6 \pm 0.01$ & 0.354 & $43.8 \pm 0.02$ & 1.044 & $41.14 \pm 0.02$ & 1.413 & $39.75 \pm 0.02$ \\
\hline 0.378 & $32.4 \pm 0.02$ & 0.386 & $42.33 \pm 0.02$ & 1.118 & $39.17 \pm 0.03$ & 1.509 & $38.78 \pm 0.03$ \\
\hline 0.405 & $30.2 \pm 0.02$ & 0.418 & $42.19 \pm 0.01$ & 1.189 & $38.38 \pm 0.02$ & 1.601 & $37.24 \pm 0.02$ \\
\hline 0.432 & $30.2 \pm 0.03$ & 0.450 & $42 \pm 0.002$ & 1.257 & $37.65 \pm 0.05$ & 1.690 & $36.17 \pm 0.03$ \\
\hline 0.458 & $30.31 \pm 0.02$ & 0.482 & $41.12 \pm 0.01$ & 1.324 & $36.55 \pm 0.05$ & 1.775 & $35.38 \pm 0.02$ \\
\hline 0.483 & $30.42 \pm 0.03$ & 0.514 & $40.18 \pm 0.02$ & 1.387 & $36.16 \pm 0.03$ & 1.856 & $34.6 \pm 0.02$ \\
\hline 0.508 & $30.9 \pm 0.03$ & 0.546 & $38.18 \pm 0.02$ & 1.449 & $36.12 \pm 0.02$ & 1.935 & $34.5 \pm 0.01$ \\
\hline 0.533 & $31.6 \pm 0.02$ & 0.579 & $36.57 \pm 0.02$ & 1.508 & $35.74 \pm 0.02$ & 2.011 & $34.94 \pm 0.03$ \\
\hline 0.557 & $32.54 \pm 0.04$ & 0.611 & $35.54 \pm 0.03$ & 1.566 & $35.44 \pm 0.03$ & 2.084 & $34.38 \pm 0.03$ \\
\hline 0.581 & $33.77 \pm 0.01$ & 0.643 & $33.64 \pm 0.02$ & 1.622 & $36.34 \pm 0.02$ & 2.154 & $35.12 \pm 0.04$ \\
\hline 0.604 & $34.15 \pm 0.02$ & 0.675 & $33.43 \pm 0.01$ & 1.676 & $37.89 \pm 0.05$ & 2.222 & $35.99 \pm 0.03$ \\
\hline 0.627 & $35.48 \pm 0.02$ & 0.707 & $33.86 \pm 0.01$ & 1.728 & $37.15 \pm 0.02$ & 2.287 & $36.44 \pm 0.05$ \\
\hline 0.649 & $34.86 \pm 0.02$ & 0.739 & $35.63 \pm 0.01$ & 1.778 & $36.1 \pm 0.03$ & 2.350 & $36.48 \pm 0.03$ \\
\hline 0.671 & $35.49 \pm 0.03$ & 0.771 & $35.72 \pm 0.02$ & 1.827 & $37.14 \pm 0.02$ & 2.411 & $36.44 \pm 0.02$ \\
\hline 0.692 & $35.44 \pm 0.03$ & 0.804 & $34.4 \pm 0.05$ & 1.875 & $36.4 \pm 0.03$ & 2.471 & $36.37 \pm 0.03$ \\
\hline 0.713 & $34.99 \pm 0.03$ & 0.836 & $35.51 \pm 0.04$ & 1.921 & $36.32 \pm 0.03$ & 2.528 & $36.15 \pm 0.03$ \\
\hline & & 0.868 & $35.6 \pm 0.03$ & 1.966 & $36.22 \pm 0.04$ & 2.583 & $35.46 \pm 0.02$ \\
\hline & & 0.900 & $34.43 \pm 0.03$ & 2.010 & $37.27 \pm 0.02$ & 2.637 & $35.16 \pm 0.01$ \\
\hline & & 0.932 & $33.4 \pm 0.02$ & 2.052 & $37.79 \pm 0.02$ & 2.689 & $34.34 \pm 0.04$ \\
\hline & & 0.964 & $33.36 \pm 0.02$ & 2.093 & $36.78 \pm 0.02$ & 2.739 & $34.52 \pm 0.03$ \\
\hline & & 0.996 & $33.58 \pm 0.02$ & 2.133 & $35.65 \pm 0.02$ & 2.788 & $35.33 \pm 0.03$ \\
\hline & & 1.029 & $33.37 \pm 0.02$ & 2.172 & $35.59 \pm 0.01$ & & \\
\hline & & 1.061 & $33.51 \pm 0.02$ & & & & \\
\hline
\end{tabular}


Supporting Information

\begin{tabular}{|c|c|c|c|c|c|c|c|}
\hline \multicolumn{8}{|c|}{ dodecyl betainate gemini $(\mathrm{s}=3)$} \\
\hline \multicolumn{2}{|c|}{ EG $(0 \%)$} & \multicolumn{2}{|c|}{ EG $(20 \%)$} & \multicolumn{2}{|c|}{ EG $(35 \%)$} & \multicolumn{2}{|c|}{$\mathrm{EG}(50 \%)$} \\
\hline $\begin{array}{c}C \\
\mathrm{mmol} \cdot \mathrm{kg}^{-1}\end{array}$ & $\begin{array}{c}\gamma \\
\mathrm{mN} \cdot \mathrm{m}^{-1}\end{array}$ & $\begin{array}{c}C \\
\mathrm{mmol} \cdot \mathrm{kg}^{-1}\end{array}$ & $\begin{array}{c}\gamma \\
\mathrm{mN} \cdot \mathrm{m}^{-1}\end{array}$ & $\begin{array}{c}C \\
\mathrm{mmol} \cdot \mathrm{kg}^{-1}\end{array}$ & $\begin{array}{c}\gamma \\
\mathrm{mN} \cdot \mathrm{m}^{-1}\end{array}$ & $\begin{array}{c}C \\
\mathrm{mmol} \cdot \mathrm{kg}^{-1}\end{array}$ & $\begin{array}{c}\gamma \\
\mathrm{mN} \cdot \mathrm{m}^{-1}\end{array}$ \\
\hline 0.024 & $64 \pm 0.02$ & 0.036 & $63.15 \pm 0.02$ & 0.071 & $57.2 \pm 0.01$ & 0.110 & $53.2 \pm 0.03$ \\
\hline 0.036 & $62.09 \pm 0.03$ & 0.059 & $60.76 \pm 0.02$ & 0.141 & $55.8 \pm 0.02$ & 0.217 & $51.8 \pm 0.03$ \\
\hline 0.059 & $60.73 \pm 0.02$ & 0.093 & $60.35 \pm 0.01$ & 0.208 & $55.22 \pm 0.02$ & 0.321 & $50.22 \pm 0.02$ \\
\hline 0.082 & $58.99 \pm 0.03$ & 0.126 & $55.45 \pm 0.02$ & 0.275 & $54.25 \pm 0.03$ & 0.421 & $49.11 \pm 0.04$ \\
\hline 0.104 & $56.45 \pm 0.02$ & 0.159 & $50.13 \pm 0.03$ & 0.340 & $54.03 \pm 0.01$ & 0.519 & $48.03 \pm 0.02$ \\
\hline 0.126 & $50.12 \pm 0.02$ & 0.191 & $47.54 \pm 0.03$ & 0.403 & $53.98 \pm 0.02$ & 0.613 & $46.98 \pm 0.03$ \\
\hline 0.148 & $47.5 \pm 0.02$ & 0.222 & $43.86 \pm 0.02$ & 0.465 & $52.87 \pm 0.03$ & 0.705 & $45.87 \pm 0.01$ \\
\hline 0.170 & $43.86 \pm 0.03$ & 0.253 & $40.2 \pm 0.03$ & 0.526 & $51.19 \pm 0.03$ & 0.794 & $43.19 \pm 0.03$ \\
\hline 0.191 & $40.2 \pm 0.02$ & 0.283 & $37.98 \pm 0.04$ & 0.585 & $50 \pm 0.03$ & 0.881 & $40.92 \pm 0.03$ \\
\hline 0.212 & $36.56 \pm 0.01$ & 0.312 & $37.64 \pm 0.04$ & 0.643 & $49.02 \pm 0.03$ & 0.986 & $40.02 \pm 0.02$ \\
\hline 0.232 & $35.24 \pm 0.01$ & 0.340 & $36.84 \pm 0.03$ & 0.700 & $48.75 \pm 0.04$ & 1.088 & $39.75 \pm 0.02$ \\
\hline 0.253 & $33.57 \pm 0.02$ & 0.368 & $35.8 \pm 0.03$ & 0.755 & $45.78 \pm 0.02$ & 1.186 & $38.78 \pm 0.02$ \\
\hline 0.273 & $31.77 \pm 0.02$ & 0.396 & $35.4 \pm 0.03$ & 0.810 & $43.24 \pm 0.02$ & 1.281 & $37.24 \pm 0.03$ \\
\hline 0.292 & $32.5 \pm 0.01$ & 0.423 & $34.78 \pm 0.01$ & 0.898 & $41 \pm 0.02$ & 1.373 & $36.17 \pm 0.02$ \\
\hline 0.312 & $32.27 \pm 0.03$ & 0.449 & $33.74 \pm 0.03$ & 0.983 & $40.38 \pm 0.01$ & 1.462 & $35.38 \pm 0.02$ \\
\hline 0.331 & $31.91 \pm 0.03$ & 0.475 & $32.9 \pm 0.04$ & 1.066 & $38.26 \pm 0.03$ & 1.548 & $34.6 \pm 0.03$ \\
\hline 0.350 & $31.56 \pm 0.04$ & 0.500 & $32.98 \pm 0.03$ & 1.114 & $35.93 \pm 0.03$ & 1.615 & $33.5 \pm 0.03$ \\
\hline 0.368 & $31.58 \pm 0.02$ & 0.525 & $32.57 \pm 0.01$ & 1.161 & $33.94 \pm 0.01$ & 1.689 & $32.94 \pm 0.04$ \\
\hline 0.387 & $31.24 \pm 0.03$ & 0.549 & $32 \pm 0.02$ & 1.208 & $32 \pm 0.01$ & 1.753 & $31.38 \pm 0.03$ \\
\hline 0.405 & $30.92 \pm 0.04$ & 0.573 & $32 \pm 0.02$ & 1.253 & $32.12 \pm 0.03$ & 1.815 & $32.12 \pm 0.02$ \\
\hline 0.423 & $30.6 \pm 0.04$ & 0.596 & $30.58 \pm 0.03$ & 1.298 & $32.99 \pm 0.02$ & 1.876 & $31.99 \pm 0.02$ \\
\hline 0.440 & $30.6 \pm 0.04$ & 0.619 & $30.26 \pm 0.05$ & 1.342 & $32.44 \pm 0.02$ & 1.935 & $31.44 \pm 0.02$ \\
\hline 0.458 & $30.26 \pm 0.03$ & 0.642 & $31.93 \pm 0.04$ & 1.385 & $34.48 \pm 0.03$ & 1.993 & $31.48 \pm 0.01$ \\
\hline 0.475 & $29.93 \pm 0.02$ & 0.664 & $30.26 \pm 0.05$ & 1.427 & $34.44 \pm 0.05$ & 2.050 & $32.44 \pm 0.02$ \\
\hline 0.492 & $30.26 \pm 0.02$ & 0.685 & $32.58 \pm 0.03$ & 1.468 & $34.37 \pm 0.05$ & 2.105 & $31.37 \pm 0.02$ \\
\hline 0.508 & $30.59 \pm 0.02$ & 0.706 & $32.9 \pm 0.03$ & 1.509 & $33.15 \pm 0.04$ & 2.159 & $31.15 \pm 0.04$ \\
\hline 0.525 & $30.9 \pm 0.04$ & 0.727 & $31.25 \pm 0.02$ & 1.549 & $33.46 \pm 0.01$ & 2.212 & $31.46 \pm 0.04$ \\
\hline 0.541 & $31.24 \pm 0.05$ & 0.748 & $31.25 \pm 0.02$ & 1.588 & $33.16 \pm 0.03$ & 2.264 & $32.16 \pm 0.03$ \\
\hline 0.557 & $31.24 \pm 0.05$ & 0.768 & $31.25 \pm 0.01$ & 1.627 & $32.34 \pm 0.02$ & 2.315 & $31.34 \pm 0.02$ \\
\hline 0.573 & $31.25 \pm 0.03$ & 0.788 & $30.58 \pm 0.02$ & 1.665 & $32.52 \pm 0.02$ & & \\
\hline 0.588 & $30.58 \pm 0.02$ & 0.807 & $29.6 \pm 0.02$ & 1.702 & $32.33 \pm 0.02$ & & \\
\hline & & & & 1.739 & $32.33 \pm 0.03$ & & \\
\hline & & & & 1.775 & $32.78 \pm 0.03$ & & \\
\hline & & & & 1.810 & $32.63 \pm 0.02$ & & \\
\hline
\end{tabular}


Supporting Information

\begin{tabular}{|c|c|c|c|c|c|c|c|}
\hline \multicolumn{8}{|c|}{ TTAB } \\
\hline \multicolumn{2}{|c|}{ EG $(0 \%)$} & \multicolumn{2}{|c|}{ EG $(20 \%)$} & \multicolumn{2}{|c|}{ EG $(35 \%)$} & \multicolumn{2}{|c|}{ EG $(50 \%)$} \\
\hline $\begin{array}{c}C \\
\mathrm{mmol} \cdot \mathrm{kg}^{-1}\end{array}$ & $\begin{array}{c}\gamma \\
\mathrm{mN} \cdot \mathrm{m}^{-1}\end{array}$ & $\begin{array}{c}C \\
\mathrm{mmol} \cdot \mathrm{kg}^{-1}\end{array}$ & $\begin{array}{c}\gamma \\
\mathrm{mN} \cdot \mathrm{m}^{-1}\end{array}$ & $\begin{array}{c}C \\
\mathrm{mmol} \cdot \mathrm{kg}^{-1}\end{array}$ & $\begin{array}{c}\gamma \\
\mathrm{mN} \cdot \mathrm{m}^{-1}\end{array}$ & $\begin{array}{c}C \\
\mathrm{mmol} \cdot \mathrm{kg}^{-1}\end{array}$ & $\begin{array}{c}\gamma \\
\mathrm{mN} \cdot \mathrm{m}^{-1}\end{array}$ \\
\hline 0.060 & $68.4 \pm 0.02$ & 0.294 & $62.04 \pm 0.05$ & 0.392 & $56.45 \pm 0.03$ & 0.588 & $56.4 \pm 0.03$ \\
\hline 0.178 & $61.39 \pm 0.02$ & 0.577 & $60.22 \pm 0.02$ & 0.989 & $54.19 \pm 0.02$ & 1.698 & $54.2 \pm 0.01$ \\
\hline 0.352 & $58.62 \pm 0.03$ & 0.849 & $57.23 \pm 0.02$ & 1.618 & $53.2 \pm 0.05$ & 2.727 & $53.2 \pm 0.02$ \\
\hline 0.632 & $56.86 \pm 0.01$ & 1.111 & $54.55 \pm 0.03$ & 2.206 & $52.45 \pm 0.03$ & 3.684 & $52.45 \pm 0.02$ \\
\hline 0.902 & $55.05 \pm 0.02$ & 1.364 & $51.5 \pm 0.04$ & 2.759 & $50.24 \pm 0.04$ & 4.490 & $50.24 \pm 0.02$ \\
\hline 1.162 & $53.82 \pm 0.03$ & 1.607 & $49.12 \pm 0.05$ & 3.278 & $48.65 \pm 0.01$ & 5.248 & $48.63 \pm 0.02$ \\
\hline 1.413 & $52.72 \pm 0.03$ & 1.888 & $47.65 \pm 0.01$ & 3.819 & $45.66 \pm 0.02$ & 6.038 & $45.56 \pm 0.01$ \\
\hline 1.655 & $50.87 \pm 0.04$ & 2.158 & $45.67 \pm 0.02$ & 4.326 & $43.23 \pm 0.02$ & 6.780 & $43.32 \pm 0.02$ \\
\hline 1.888 & $46.83 \pm 0.02$ & 2.416 & $43.11 \pm 0.03$ & 4.756 & $42.81 \pm 0.02$ & 7.410 & $42.79 \pm 0.02$ \\
\hline 2.113 & $45.06 \pm 0.02$ & 2.664 & $41.51 \pm 0.05$ & 5.163 & $41.58 \pm 0.01$ & 8.006 & $41.6 \pm 0.03$ \\
\hline 2.331 & $43.45 \pm 0.02$ & 2.903 & $40.32 \pm 0.02$ & 5.549 & $40.69 \pm 0.03$ & 8.571 & $40.6 \pm 0.02$ \\
\hline 2.542 & $41.47 \pm 0.01$ & 3.133 & $38.9 \pm 0.02$ & 5.915 & $39.48 \pm 0.03$ & 9.109 & $39.48 \pm 0.04$ \\
\hline 2.745 & $40.66 \pm 0.03$ & 3.354 & $38.17 \pm 0.03$ & 6.264 & $38.97 \pm 0.03$ & 9.620 & $38.89 \pm 0.02$ \\
\hline 2.942 & $39.9 \pm 0.03$ & 3.567 & $37.45 \pm 0.01$ & 6.523 & $38.55 \pm 0.02$ & 10.053 & $38.44 \pm 0.02$ \\
\hline 3.133 & $38.87 \pm 0.04$ & 3.872 & $36.5 \pm 0.02$ & 6.772 & $38.15 \pm 0.02$ & 10.469 & $38.15 \pm 0.02$ \\
\hline 3.318 & $37.45 \pm 0.02$ & 4.162 & $35.98 \pm 0.02$ & 7.013 & $37.95 \pm 0.04$ & 10.867 & $37.95 \pm 0.03$ \\
\hline 3.497 & $36.48 \pm 0.02$ & 4.347 & $35.66 \pm 0.02$ & 7.245 & $37.64 \pm 0.05$ & 11.203 & $37.58 \pm 0.05$ \\
\hline 3.671 & $35.45 \pm 0.02$ & 4.641 & $34.25 \pm 0.03$ & 7.437 & $37.14 \pm 0.05$ & 11.481 & $37.23 \pm 0.03$ \\
\hline 3.839 & $35.17 \pm 0.02$ & 4.919 & $34.17 \pm 0.02$ & 7.624 & $36.46 \pm 0.03$ & 11.752 & $36.47 \pm 0.04$ \\
\hline 4.003 & $35.23 \pm 0.01$ & 5.183 & $34.22 \pm 0.05$ & 7.864 & $36.45 \pm 0.03$ & 12.100 & $36.39 \pm 0.04$ \\
\hline 4.162 & $35.46 \pm 0.02$ & 5.434 & $34.42 \pm 0.03$ & 8.095 & $36.46 \pm 0.01$ & 12.436 & $36.46 \pm 0.02$ \\
\hline 4.316 & $35.34 \pm 0.02$ & 5.672 & $34.67 \pm 0.04$ & 8.318 & $36.65 \pm 0.02$ & 12.759 & $36.65 \pm 0.03$ \\
\hline 4.466 & $35.25 \pm 0.03$ & 5.898 & $35.05 \pm 0.04$ & 8.532 & $36.71 \pm 0.01$ & 13.070 & $36.69 \pm 0.03$ \\
\hline 4.612 & $35.28 \pm 0.04$ & 6.114 & $34.73 \pm 0.02$ & 8.739 & $36.65 \pm 0.02$ & 13.370 & $36.65 \pm 0.03$ \\
\hline 4.754 & $35.11 \pm 0.03$ & & & 8.938 & $36.71 \pm 0.02$ & 13.660 & $36.73 \pm 0.02$ \\
\hline
\end{tabular}


Supporting Information

Table S2. The specific conductivities $(\kappa)$ and corresponding concentrations $(C)$ of dodecyl esterquat gemini ( $(s=3)$, dodecyl betainate gemini $(s=3)$, and TTAB in the water-EG medium at $\mathrm{T}=298.15 \mathrm{~K}$

\begin{tabular}{|c|c|c|c|c|c|c|c|}
\hline \multicolumn{8}{|c|}{ dodecyl esterquat gemini $(\mathrm{s}=3)$} \\
\hline \multicolumn{2}{|c|}{$\mathrm{EG}(0 \%)$} & \multicolumn{2}{|c|}{ EG $(20 \%)$} & \multicolumn{2}{|c|}{$\mathrm{EG}(35 \%)$} & \multicolumn{2}{|c|}{ EG $(50 \%)$} \\
\hline $\begin{array}{c}C \\
\mathrm{mmol} \cdot \mathrm{kg}^{-1}\end{array}$ & $\begin{array}{c}\kappa \\
\mu \mathrm{S} \cdot \mathrm{cm}^{-1}\end{array}$ & $\begin{array}{c}C \\
\mathrm{mmol} \cdot \mathrm{kg}^{-1}\end{array}$ & $\begin{array}{c}\kappa \\
\mu \mathrm{S} \cdot \mathrm{cm}^{-1}\end{array}$ & $\begin{array}{c}C \\
\mathrm{mmol} \cdot \mathrm{kg}^{-1}\end{array}$ & $\begin{array}{c}\kappa \\
\mu \mathrm{S} \cdot \mathrm{cm}^{-1}\end{array}$ & $\begin{array}{c}C \\
\mathrm{mmol} \cdot \mathrm{kg}^{-1}\end{array}$ & $\begin{array}{c}\kappa \\
\mu \mathrm{S} \cdot \mathrm{cm}^{-1}\end{array}$ \\
\hline 0.000 & $2.66 \pm 0.10$ & 0.060 & $12.5 \pm 0.22$ & 0.120 & $10 \pm 0.23$ & 0.209 & $7 \pm 0.35$ \\
\hline 0.036 & $6.8 \pm 0.25$ & 0.120 & $15.3 \pm 0.25$ & 0.238 & $14 \pm 0.25$ & 0.414 & $12 \pm 0.33$ \\
\hline 0.060 & $14.3 \pm 0.16$ & 0.179 & $19.5 \pm 0.26$ & 0.356 & $20 \pm 0.24$ & 0.617 & $16.5 \pm 0.31$ \\
\hline 0.078 & $19.5 \pm 0.18$ & 0.238 & $25.6 \pm 0.31$ & 0.472 & $25 \pm 0.25$ & 0.817 & $24 \pm 0.34$ \\
\hline 0.096 & $25.6 \pm 0.19$ & 0.297 & $32 \pm 0.36$ & 0.588 & $32.1 \pm 0.26$ & 1.014 & $30.3 \pm 0.31$ \\
\hline 0.114 & $32 \pm 0.15$ & 0.356 & $38.3 \pm 0.44$ & 0.703 & $37.1 \pm 0.17$ & 1.209 & $36.4 \pm 0.32$ \\
\hline 0.131 & $35.7 \pm 0.14$ & 0.414 & $44.3 \pm 0.46$ & 0.817 & $43 \pm 0.15$ & 1.401 & $41 \pm 0.25$ \\
\hline 0.149 & $44 \pm 0.14$ & 0.472 & $50.2 \pm 0.28$ & 0.930 & $49.1 \pm 0.22$ & 1.591 & $46 \pm 0.26$ \\
\hline 0.167 & $51 \pm 0.23$ & 0.530 & $55 \pm 0.20$ & 1.042 & $53.2 \pm 0.34$ & 1.778 & $51 \pm 0.32$ \\
\hline 0.185 & $58 \pm 0.28$ & 0.588 & $60.3 \pm 0.23$ & 1.154 & $59.1 \pm 0.31$ & 1.963 & $58.5 \pm 0.28$ \\
\hline 0.203 & $68.6 \pm 0.31$ & 0.646 & $63.8 \pm 0.15$ & 1.264 & $64 \pm 0.24$ & 2.145 & $60 \pm 0.32$ \\
\hline 0.220 & $74 \pm 0.26$ & 0.703 & $66 \pm 0.18$ & 1.374 & $67 \pm 0.25$ & 2.325 & $62.1 \pm 0.35$ \\
\hline 0.238 & $80.6 \pm 0.34$ & 0.760 & $69.3 \pm 0.14$ & 1.483 & $69 \pm 0.21$ & 2.502 & $64.7 \pm 0.26$ \\
\hline 0.256 & $87.8 \pm 0.36$ & 0.817 & $72.7 \pm 0.15$ & 1.591 & $73 \pm 0.26$ & 2.678 & $67 \pm 0.25$ \\
\hline 0.273 & $96 \pm 0.35$ & 0.874 & $75.7 \pm 0.15$ & 1.698 & $75 \pm 0.16$ & 2.851 & $71 \pm 0.25$ \\
\hline 0.297 & $102.8 \pm 0.41$ & 0.930 & $77.9 \pm 0.16$ & 1.805 & $76.9 \pm 0.23$ & 3.022 & $73.5 \pm 0.26$ \\
\hline 0.315 & $108.6 \pm 0.22$ & 0.986 & $80.5 \pm 0.14$ & 1.910 & $79.5 \pm 0.33$ & 3.190 & $74.5 \pm 0.20$ \\
\hline 0.332 & $111.6 \pm 0.35$ & 1.042 & $83.5 \pm 0.21$ & 2.015 & $83 \pm 0.27$ & 3.357 & $80.1 \pm 0.31$ \\
\hline 0.350 & $114.8 \pm 0.26$ & 1.098 & $85 \pm 0.23$ & 2.119 & $85 \pm 0.28$ & 3.522 & $83 \pm 0.33$ \\
\hline 0.367 & $117.2 \pm 0.24$ & 1.154 & $88.4 \pm 0.24$ & 2.222 & $88 \pm 0.42$ & 3.684 & $84 \pm 0.42$ \\
\hline 0.385 & $120.6 \pm 0.22$ & 1.209 & $90.1 \pm 0.36$ & 2.325 & $90 \pm 0.45$ & 3.845 & $85 \pm 0.44$ \\
\hline 0.403 & $124.8 \pm 0.21$ & 1.264 & $92.3 \pm 0.33$ & 2.426 & $92 \pm 0.40$ & 4.003 & $85.6 \pm 0.46$ \\
\hline 0.420 & $126.8 \pm 0.26$ & 1.319 & $94 \pm 0.18$ & 2.527 & $93.5 \pm 0.35$ & 4.160 & $89.7 \pm 0.36$ \\
\hline 0.438 & $128.4 \pm 0.23$ & 1.374 & $96 \pm 0.43$ & 2.628 & $96.5 \pm 0.21$ & 4.315 & $93.3 \pm 0.34$ \\
\hline 0.455 & $130.6 \pm 0.38$ & 1.429 & $98 \pm 0.51$ & 2.727 & $97.9 \pm 0.34$ & 4.468 & $95.3 \pm 0.34$ \\
\hline 0.472 & $132 \pm 0.42$ & 1.483 & $100 \pm 0.42$ & 2.826 & $100 \pm 0.22$ & 4.619 & $98.7 \pm 0.32$ \\
\hline 0.490 & $134.2 \pm 0.35$ & 1.537 & $103 \pm 0.35$ & 2.924 & $101 \pm 0.20$ & & \\
\hline 0.507 & $136 \pm 0.27$ & 1.591 & $104.3 \pm 0.35$ & 3.022 & $103 \pm 0.21$ & & \\
\hline 0.525 & $138.2 \pm 0.25$ & 1.645 & $105.2 \pm 0.34$ & & & & \\
\hline 0.542 & $138.8 \pm 0.23$ & 1.698 & $107 \pm 0.32$ & & & & \\
\hline
\end{tabular}




\section{Supporting Information}

\begin{tabular}{|c|c|c|c|c|c|c|c|}
\hline \multicolumn{8}{|c|}{ dodecyl betainate gemini $(\mathrm{s}=3)$} \\
\hline \multicolumn{2}{|c|}{$\mathrm{EG}(0 \%)$} & \multicolumn{2}{|c|}{ EG $(20 \%)$} & \multicolumn{2}{|c|}{ EG $(35 \%)$} & \multicolumn{2}{|c|}{ EG $(50 \%)$} \\
\hline $\begin{array}{c}C \\
\mathrm{mmol} \cdot \mathrm{kg}^{-1}\end{array}$ & $\begin{array}{c}\kappa \\
\mu \mathrm{S} \cdot \mathrm{cm}^{-1}\end{array}$ & $\begin{array}{c}C \\
\mathrm{mmol} \cdot \mathrm{kg}^{-1}\end{array}$ & $\begin{array}{c}\kappa \\
\mu \mathrm{S} \cdot \mathrm{cm}^{-1}\end{array}$ & $\begin{array}{c}C \\
\mathrm{mmol} \cdot \mathrm{kg}^{-1}\end{array}$ & $\begin{array}{c}\kappa \\
\mu \mathrm{S} \cdot \mathrm{cm}^{-1}\end{array}$ & $\begin{array}{c}C \\
\mathrm{mmol} \cdot \mathrm{kg}^{-1}\end{array}$ & $\begin{array}{c}\kappa \\
\mu \mathrm{S} \cdot \mathrm{cm}^{-1}\end{array}$ \\
\hline 0.000 & $1.88 \pm 0.15$ & 0.119 & $15.8 \pm 0.23$ & 0.179 & $13 \pm 0.12$ & 0.348 & $10 \pm 0.31$ \\
\hline 0.020 & $12.3 \pm 0.21$ & 0.178 & $20.5 \pm 0.25$ & 0.356 & $20 \pm 0.15$ & 0.690 & $18 \pm 0.33$ \\
\hline 0.030 & $17.1 \pm 0.23$ & 0.265 & $31.3 \pm 0.34$ & 0.530 & $26 \pm 0.15$ & 1.028 & $26 \pm 0.16$ \\
\hline 0.045 & $22.2 \pm 0.25$ & 0.352 & $40.1 \pm 0.46$ & 0.703 & $34 \pm 0.20$ & 1.362 & $34 \pm 0.25$ \\
\hline 0.060 & $28 \pm 0.31$ & 0.437 & $47.5 \pm 0.44$ & 0.874 & $39 \pm 0.22$ & 1.691 & $41 \pm 0.27$ \\
\hline 0.075 & $32 \pm 0.26$ & 0.521 & $51.6 \pm 0.52$ & 1.042 & $43 \pm 0.17$ & 2.015 & $46 \pm 0.24$ \\
\hline 0.089 & $36.5 \pm 0.35$ & 0.605 & $53.2 \pm 0.50$ & 1.209 & $51 \pm 0.25$ & 2.336 & $49.7 \pm 0.24$ \\
\hline 0.104 & $40 \pm 0.34$ & 0.687 & $57.2 \pm 0.44$ & 1.374 & $53 \pm 0.26$ & 2.652 & $52.7 \pm 0.18$ \\
\hline 0.119 & $44.3 \pm 0.34$ & 0.769 & $60.2 \pm 0.46$ & 1.537 & $55.7 \pm 0.31$ & 2.963 & $56.5 \pm 0.26$ \\
\hline 0.134 & $49.5 \pm 0.15$ & 0.849 & $63.9 \pm 0.31$ & 1.698 & $61 \pm 0.33$ & 3.271 & $60 \pm 0.25$ \\
\hline 0.149 & $53.4 \pm 0.18$ & 0.929 & $67.4 \pm 0.27$ & 1.857 & $63.4 \pm 0.26$ & 3.575 & $63.1 \pm 0.28$ \\
\hline 0.163 & $57.5 \pm 0.26$ & 1.007 & $70.1 \pm 0.25$ & 2.015 & $66.2 \pm 0.24$ & 3.875 & $65 \pm 0.35$ \\
\hline 0.178 & $63 \pm 0.16$ & 1.085 & $73.6 \pm 0.22$ & 2.171 & $68 \pm 0.23$ & 4.170 & $70 \pm 0.31$ \\
\hline 0.192 & $68.8 \pm 0.24$ & 1.162 & $77.7 \pm 0.23$ & 2.325 & $71 \pm 0.35$ & 4.463 & $74 \pm 0.38$ \\
\hline 0.207 & $73.5 \pm 0.25$ & 1.239 & $82 \pm 0.25$ & 2.477 & $75.5 \pm 0.16$ & 4.751 & $77 \pm 0.32$ \\
\hline 0.222 & $80 \pm 0.33$ & 1.314 & $85.5 \pm 0.25$ & 2.628 & $77.8 \pm 0.43$ & 5.036 & $79 \pm 0.29$ \\
\hline 0.236 & $86.5 \pm 0.25$ & 1.388 & $88.5 \pm 0.18$ & 2.777 & $80.7 \pm 0.27$ & 5.317 & $82 \pm 0.30$ \\
\hline 0.251 & $88.8 \pm 0.24$ & 1.462 & $90 \pm 0.31$ & 2.924 & $84 \pm 0.29$ & 5.595 & $84.9 \pm 0.42$ \\
\hline 0.265 & $91 \pm 0.25$ & 1.535 & $93 \pm 0.25$ & 3.070 & $87 \pm 0.27$ & 5.869 & $88 \pm 0.43$ \\
\hline 0.280 & $94 \pm 0.25$ & 1.607 & $97 \pm 0.36$ & 3.214 & $90.2 \pm 0.25$ & 6.140 & $93 \pm 0.37$ \\
\hline 0.294 & $96.2 \pm 0.24$ & 1.679 & $99 \pm 0.32$ & 3.357 & $91.4 \pm 0.25$ & 6.408 & $96.5 \pm 0.35$ \\
\hline 0.309 & $99 \pm 0.28$ & 1.749 & $104 \pm 0.32$ & 3.498 & $93.1 \pm 0.25$ & 6.672 & $100.4 \pm 0.35$ \\
\hline 0.323 & $100.1 \pm 0.30$ & 1.819 & $105.9 \pm 0.19$ & 3.638 & $94 \pm 0.24$ & 6.934 & $103.9 \pm 0.29$ \\
\hline 0.337 & $102.5 \pm 0.28$ & 1.888 & $110.5 \pm 0.20$ & 3.776 & $96.3 \pm 0.26$ & 7.192 & $108 \pm 0.33$ \\
\hline 0.352 & $103.4 \pm 0.33$ & 1.957 & $115 \pm 0.23$ & 3.913 & $98.9 \pm 0.30$ & 7.447 & $111 \pm 0.31$ \\
\hline 0.366 & $104.5 \pm 0.36$ & & & 4.048 & $100.5 \pm 0.34$ & 7.699 & $115 \pm 0.36$ \\
\hline
\end{tabular}




\begin{tabular}{|c|c|c|c|c|c|c|c|}
\hline \multicolumn{8}{|c|}{ TTAB } \\
\hline \multicolumn{2}{|c|}{ EG $(0 \%)$} & \multicolumn{2}{|c|}{ EG $(20 \%)$} & \multicolumn{2}{|c|}{ EG $(35 \%)$} & \multicolumn{2}{|c|}{ EG $(50 \%)$} \\
\hline $\begin{array}{c}C \\
\mathrm{mmol} \cdot \mathrm{kg}^{-1}\end{array}$ & $\begin{array}{c}\kappa \\
\mu \mathrm{S} \cdot \mathrm{cm}^{-1}\end{array}$ & $\begin{array}{c}C \\
\mathrm{mmol} \cdot \mathrm{kg}^{-1}\end{array}$ & $\begin{array}{c}\kappa \\
\mu \mathrm{S} \cdot \mathrm{cm}^{-1}\end{array}$ & $\begin{array}{c}C \\
\mathrm{mmol} \cdot \mathrm{kg}^{-1}\end{array}$ & $\begin{array}{c}\kappa \\
\mu \mathrm{S} \cdot \mathrm{cm}^{-1}\end{array}$ & $\begin{array}{c}C \\
\mathrm{mmol} \cdot \mathrm{kg}^{-1}\end{array}$ & $\begin{array}{c}\kappa \\
\mu \mathrm{S} \cdot \mathrm{cm}^{-1}\end{array}$ \\
\hline 0.000 & $1.71 \pm 0.22$ & 0.495 & $30 \pm 0.31$ & 0.792 & $37.2 \pm 0.17$ & 1.485 & $35.66 \pm 0.26$ \\
\hline 0.396 & $45.5 \pm 0.26$ & 0.980 & $50 \pm 0.35$ & 1.569 & $68 \pm 0.19$ & 2.941 & $64.3 \pm 0.26$ \\
\hline 0.784 & $70 \pm 0.25$ & 1.456 & $90 \pm 0.36$ & 2.330 & $115 \pm 0.26$ & 4.369 & $104.2 \pm 0.33$ \\
\hline 1.165 & $114 \pm 0.16$ & 1.923 & $127 \pm 0.25$ & 3.077 & $150 \pm 0.24$ & 5.769 & $144 \pm 0.38$ \\
\hline 1.538 & $140 \pm 0.25$ & 2.381 & $170 \pm 0.24$ & 3.810 & $177.7 \pm 0.35$ & 7.143 & $178 \pm 0.24$ \\
\hline 1.905 & $180 \pm 0.24$ & 2.830 & $200 \pm 0.32$ & 4.528 & $220 \pm 0.33$ & 8.491 & $216 \pm 0.35$ \\
\hline 2.264 & $220 \pm 0.36$ & 3.271 & $240 \pm 0.18$ & 5.234 & $255 \pm 0.35$ & 9.813 & $258 \pm 0.24$ \\
\hline 2.617 & $260 \pm 0.34$ & 3.704 & $280 \pm 0.26$ & 5.926 & $281 \pm 0.18$ & 11.111 & $290 \pm 0.27$ \\
\hline 2.963 & $294 \pm 0.38$ & 4.128 & $312 \pm 0.24$ & 6.606 & $310 \pm 0.24$ & 12.385 & $318 \pm 0.28$ \\
\hline 3.303 & $327 \pm 0.44$ & 4.545 & $340 \pm 0.22$ & 7.273 & $334 \pm 0.26$ & 13.636 & $322 \pm 0.15$ \\
\hline 3.636 & $355 \pm 0.37$ & 4.955 & $367 \pm 0.31$ & 7.928 & $349 \pm 0.24$ & 14.865 & $328 \pm 0.17$ \\
\hline 3.964 & $385 \pm 0.45$ & 5.357 & $370 \pm 0.35$ & 8.571 & $356 \pm 0.33$ & 16.071 & $339 \pm 0.33$ \\
\hline 4.286 & $395 \pm 0.45$ & 5.752 & $382 \pm 0.41$ & 9.204 & $365.8 \pm 0.30$ & 17.257 & $342 \pm 0.44$ \\
\hline 4.602 & $410 \pm 0.52$ & 6.140 & $389 \pm 0.46$ & 9.825 & $376 \pm 0.25$ & 18.421 & $352 \pm 0.32$ \\
\hline 4.912 & $422 \pm 0.41$ & 6.522 & $396 \pm 0.38$ & 10.435 & $381 \pm 0.26$ & 19.565 & $363 \pm 0.31$ \\
\hline 5.217 & $434 \pm 0.33$ & 6.897 & $403 \pm 0.33$ & 11.034 & $386 \pm 0.25$ & 20.690 & $376 \pm 0.33$ \\
\hline 5.517 & $439 \pm 0.35$ & 7.265 & $410 \pm 0.25$ & 11.624 & $393 \pm 0.46$ & 21.795 & $386 \pm 0.33$ \\
\hline 5.812 & $447 \pm 0.34$ & 7.627 & $420 \pm 0.24$ & 12.203 & $401 \pm 0.48$ & 22.881 & $393 \pm 0.34$ \\
\hline 6.102 & $458 \pm 0.35$ & 7.983 & $428 \pm 0.26$ & 12.773 & $410 \pm 0.53$ & 23.950 & $400 \pm 0.35$ \\
\hline 6.387 & $462 \pm 0.35$ & 8.333 & $437 \pm 0.26$ & 13.333 & $418.7 \pm 0.51$ & 25.000 & $416 \pm 0.31$ \\
\hline 6.667 & $473 \pm 0.30$ & 8.678 & $445 \pm 0.25$ & 13.884 & $426 \pm 0.50$ & 26.033 & $423 \pm 0.28$ \\
\hline 6.942 & $482 \pm 0.32$ & 9.016 & $452 \pm 0.27$ & 14.426 & $431 \pm 0.38$ & 27.049 & $430 \pm 0.29$ \\
\hline 7.213 & $487 \pm 0.26$ & 9.350 & $458 \pm 0.25$ & 14.959 & $441 \pm 0.33$ & 28.049 & $437 \pm 0.35$ \\
\hline 7.480 & $490 \pm 0.28$ & 9.677 & $463 \pm 0.36$ & 15.484 & $449 \pm 0.33$ & 29.032 & $444 \pm 0.35$ \\
\hline 7.742 & $492 \pm 0.32$ & 10.000 & $469 \pm 0.22$ & 16.000 & $455 \pm 0.34$ & 30.000 & $451 \pm 0.24$ \\
\hline 8.000 & $495 \pm 0.30$ & 10.317 & $478 \pm 0.24$ & 16.508 & $462 \pm 0.33$ & 30.952 & $460 \pm 0.22$ \\
\hline 8.254 & $499 \pm 0.38$ & 10.630 & $483 \pm 0.25$ & 17.008 & $467 \pm 0.36$ & 31.890 & $469 \pm 0.21$ \\
\hline 8.504 & $502 \pm 0.35$ & 10.938 & $490 \pm 0.25$ & 17.500 & $473 \pm 0.29$ & 32.813 & $478 \pm 0.22$ \\
\hline 8.750 & $504 \pm 0.35$ & 11.240 & $495 \pm 0.24$ & 17.984 & $479 \pm 0.27$ & 33.721 & $488 \pm 0.36$ \\
\hline 8.992 & $508 \pm 0.34$ & & & 18.462 & $484 \pm 0.31$ & 34.615 & $504 \pm 0.35$ \\
\hline & & & & 18.931 & $489 \pm 0.26$ & 35.496 & $512 \pm 0.43$ \\
\hline & & & & 19.394 & $496 \pm 0.25$ & 36.364 & $521 \pm 0.38$ \\
\hline & & & & & & 37.218 & $531 \pm 0.35$ \\
\hline & & & & & & 38.060 & $541 \pm 0.36$ \\
\hline
\end{tabular}


Supporting Information

Table S3. The relative viscosity $\left(\eta / \eta_{0}\right)$ and corresponding concentrations $(C)$ at $0 \%$ and $35 \%$ EG for dodecyl esterquat gemini $(\mathrm{s}=3)$ and dodecyl betainate gemini $(\mathrm{s}=3)$ at $\mathrm{T}=\mathbf{2 9 8 . 1 5} \mathrm{K}$

\begin{tabular}{|c|c|c|c|c|c|c|c|}
\hline \multicolumn{4}{|c|}{ dodecyl esterquat gemini $(s=3)$} & \multicolumn{4}{|c|}{ dodecyl betainate gemini $(s=3)$} \\
\hline \multicolumn{2}{|c|}{$\mathrm{EG}(0 \%)$} & \multicolumn{2}{|c|}{ EG (35\%) } & \multicolumn{2}{|c|}{$\mathrm{EG}(0 \%)$} & \multicolumn{2}{|c|}{ EG (35\%) } \\
\hline $\begin{array}{c}C \\
\mathrm{mmol} \cdot \mathrm{kg}^{-1}\end{array}$ & $\eta / \eta_{0}$ & $\begin{array}{c}C \\
\mathrm{mmol} \cdot \mathrm{kg}^{-1}\end{array}$ & $\eta / \eta_{0}$ & $\begin{array}{c}C \\
\mathrm{mmol} \cdot \mathrm{kg}^{-1}\end{array}$ & $\eta / \eta_{0}$ & $\begin{array}{c}C \\
\mathrm{mmol} \cdot \mathrm{kg}^{-1}\end{array}$ & $\eta / \eta_{0}$ \\
\hline 50 & $4.360 \pm 0.05$ & 50 & $2.579 \pm 0.03$ & 50 & $5.483 \pm 0.04$ & 50 & $2.774 \pm 0.02$ \\
\hline 40 & $2.178 \pm 0.03$ & 40 & $1.837 \pm 0.02$ & 35 & $1.604 \pm 0.02$ & 40 & $1.539 \pm 0.04$ \\
\hline 30 & $1.043 \pm 0.05$ & 30 & $1.301 \pm 0.05$ & 27 & $1.356 \pm 0.05$ & 30 & $1.345 \pm 0.02$ \\
\hline 15 & $1.121 \pm 0.01$ & 25 & $1.323 \pm 0.02$ & 20 & $1.130 \pm 0.02$ & 25 & $1.450 \pm 0.03$ \\
\hline 10 & $1.096 \pm 0.01$ & 20 & $1.311 \pm 0.03$ & 12.8 & $1.119 \pm 0.03$ & 20 & $1.272 \pm 0.01$ \\
\hline 7 & $1.056 \pm 0.01$ & 15 & $1.174 \pm 0.03$ & 10 & $1.124 \pm 0.02$ & 15 & $1.362 \pm 0.01$ \\
\hline 5 & $1.033 \pm 0.02$ & 10 & $1.311 \pm 0.02$ & 5 & $1.103 \pm 0.02$ & 10 & $1.392 \pm 0.03$ \\
\hline 3 & $1.022 \pm 0.01$ & 5 & $1.255 \pm 0.03$ & 3 & $1.091 \pm 0.02$ & 5 & $1.130 \pm 0.02$ \\
\hline 0.5 & $1.034 \pm 0.01$ & 0 & $1.000 \pm 0.01$ & 0.15 & $1.061 \pm 0.01$ & 0 & $1.000 \pm 0.01$ \\
\hline 0 & $1.000 \pm 0.00$ & & & 0 & $1.000 \pm 0.00$ & & \\
\hline
\end{tabular}

Table S4. Relative changes in cmc values and degree of counter-ion dissociation $(\alpha)$ in gemini surfactants compared to water

\begin{tabular}{|c|c|c|c|c|c|c|}
\hline \multirow{2}{*}{ surfactants } & \multicolumn{5}{|c|}{ cmc } & \multicolumn{2}{c|}{ degree of counter-ion dissociation $(\alpha)$} \\
\cline { 2 - 7 } & $0 \%$ EG & $20 \%$ EG & $50 \%$ EG & $0 \%$ EG & $20 \%$ EG & $50 \%$ EG \\
\hline dodecyl esterquat gemini (s=3) & 0.41 & $0.58(41 \%)^{\mathrm{d}}$ & $1.93(370 \%)^{\mathrm{d}}$ & 0.36 & 0.43 & 0.48 \\
\hline dodecyl betainate gemini (s=3) & 0.27 & $0.54(100 \%)^{\mathrm{d}}$ & $1.75(548 \%)^{\mathrm{d}}$ & 0.42 & 0.47 & 0.54 \\
\hline $14-2-14^{\mathrm{a}}$ & 0.151 & $0.235(55 \%)^{\mathrm{d}}$ & - & 0.25 & 0.22 & - \\
\hline $14-4-14^{\mathrm{b}}$ & 0.156 & $0.273(75 \%)^{\mathrm{d}}$ & $1.11(611 \%)^{\mathrm{d}}$ & 0.34 & 0.33 & 0.36 \\
\hline $14-5-14^{\mathrm{b}}$ & 0.168 & $0.293(74 \%)^{\mathrm{d}}$ & $1.14(578 \%)^{\mathrm{d}}$ & 0.36 & 0.35 & 0.37 \\
\hline $14-6-14^{\mathrm{b}}$ & 0.190 & $0.303(59 \%)^{\mathrm{d}}$ & $1.195(528 \%)^{\mathrm{d}}$ & 0.38 & 0.40 & 0.41 \\
\hline $12-3-12^{\mathrm{c}}$ & 0.97 & $1.26(29 \%)^{\mathrm{d}}$ & $3.63(274 \%)^{\mathrm{d}}$ & 0.21 & 0.22 & 0.24 \\
\hline
\end{tabular}

b 2

c 3

${ }^{d}$ The values in parentheses show cmc changes compared to water

(1) Sood, A.; Kaur, R.; Banipal, T., Influence of organic solvents, head-groups and temperature on the micellization behavior of some cationic surfactants. Indian J. Chem. 2016. 
Supporting Information

(2) Koya, P. A.; Wagay, T. A.; Ismail, K., Effect of ethylene glycol and propylene glycol on the aggregation of alkanediyl- $\alpha, \omega$-bis (tetradecyldimethylammonium bromide)(14-s-14; $s=4-6)$ gemini surfactants. J. Mol. Liq. 2016, 219, 505-512.

(3) Rodríguez, A.; del Mar Graciani, M.; Muñoz, M.; Robina, I.; Moyá, M. L., Effects of ethylene glycol addition on the aggregation and micellar growth of gemini surfactants. Langmuir 2006, 22, 9519-9525. 